\title{
Anomalies in the Allen and Hubbard Parallel Analysis Procedure
}

\author{
Albert A. Cota \\ University of Toronto \\ R. Stewart Longman, Ronald R. Holden, and G. Cynthia Fekken \\ Queen's University
}

\begin{abstract}
Allen and Hubbard (1986) increased the accessibility of parallel analysis for principal components applications by developing an equation to predict mean eigenvalues of random data matrices with unities in the diagonals. Two recent studies have shown, however, that Allen and Hubbard's procedure may yield degenerate solutions-that is, solutions in which succeeding eigenvalues are larger
\end{abstract}

\author{
than preceding eigenvalues. The parameters of \\ sample size and number of variables within which \\ the Allen and Hubbard equation degenerates are \\ documented. Implications for the use of this \\ procedure are discussed. Index terms: exploratory \\ factor analysis, factor extraction, parallel analysis, \\ principal components analysis, rules for factor \\ retention.
}

Determining the "correct" number of components to retain in principal components analysis is arguably the most important decision that a researcher makes (Velicer \& Jackson, 1990), because extracting too many or too few factors will have a major impact on subsequent interpretation of the analysis (Zwick \& Velicer, 1986). Numerous methods have been developed for selecting the number of components to retain in principal components analysis. Of these methods, empirical research (e.g., Zwick \& Velicer, 1986) has favored parallel analysis (Horn, 1965), which can be viewed as a correction for sample size to Kaiser's (1960) eigenvalue greater-than-one rule. Horn's procedure suggests retention of only those components from observed data with eigenvalues larger than the corresponding eigenvalues from random data. Parallel analysis is especially useful when overextraction is likely using Kaiser's rule (e.g., principal components analysis of item responses to personality questionnaires; Loehlin, 1987, p. 146).

Allen and Hubbard (1986) increased the accessibility of parallel analysis for principal components applications by developing an equation to predict mean eigenvalues of random data matrices with unities in the diagonals. Their equation, which yields up to 48 latent roots, took this form:

$\log _{e}\left(\lambda_{i}\right)=a_{i}+b_{i} \log _{e}(n-1)+c_{i} \log _{e}[(p-i-1)(p-i+2) / 2]+d_{i} \log _{e}\left(\lambda_{i-1}\right)$

where $\lambda$ is the eigenvalue, $i$ is the ordinal position of the eigenvalue; $n$ is the sample size; $p$ is the number of variables; $b_{i}, c_{i}$, and $d_{i}$ are regression coefficients; and $a_{i}$ is the intercept. For their study, $p$ ranged from 5 to 50 , and $n$ ranged from 30 to 1,000. $n$ and $p$ combinations with $n>3 p / 2$ were included in regression analyses. Predictions of mean eigenvalues using Equation 1 yielded multiple correlations of at least .965. Equation 1 has been implemented for microcomputers by Hays (1987), and Holden, Longman, Cota, and Fekken (1989). The present study investigated reports by Lautenschlager (1989), and Longman, Cota, Holden, and Fekken (1989) that the Allen and Hubbard equation

APPLIED PSYCHOLOGICAL MEASUREMENT

Vol. 15, No. 1, March 1991, pp. 95-97

(C) Copyright 1991 Applied Psychological Measurement Inc.

0146-6216/91/010095-03\$1.40 
"degenerates" (i.e., yields solutions in which eigenvalues begin to increase after a specific ordinal position) for parameter values within the range of those used in its development.

\section{Method}

Using Allen and Hubbard's (1986) equation as implemented by Holden et al. (1989), solutions of predicted mean eigenvalues for values of $p$ between 5 and 50 and several values of $n$ ranging from 30 to 1,000 were generated. $n=30$ was the lowest value used for values of $p$ from 5 to 19 . For the remaining values of $p, 76$ was the lowest value of $n$ that was used. Solutions were examined for degeneracy, with degeneracy defined as the situation where any successive eigenvalue was larger

Table 1

Ordinal Positions of Eigenvalues That Mark Onset of Degeneracy for Five Specific $n$ Values at Values of $p$ From 5 to 50

$\left(n_{\max }=\right.$ Sample Size)

\begin{tabular}{lcccccc}
\hline \hline & \multicolumn{5}{c}{ Value of $n$} \\
\cline { 3 - 7 }$p$ & $n_{\max }$ & $n_{\max }+1$ & 400 & 600 & 800 & 1,000 \\
\hline $5-15$ & - & - & - & - & - & - \\
16 & 745 & 13 & - & - & 13 & 13 \\
17 & 628 & 14 & - & - & 14 & 13 \\
18 & 707 & 14 & - & - & 14 & 13 \\
19 & 640 & 16 & - & - & 14 & 13 \\
20 & 677 & 17 & - & - & 14 & 13 \\
21 & 673 & 18 & - & - & 14 & 13 \\
22 & 657 & 19 & - & - & 14 & 12 \\
23 & 605 & 20 & - & - & 14 & 11 \\
24 & 611 & 20 & - & - & 13 & 11 \\
25 & 601 & 20 & - & - & 13 & 10 \\
26 & 586 & 20 & - & 20 & 12 & 9 \\
27 & 499 & 24 & - & 16 & 12 & 8 \\
28 & 493 & 25 & - & 16 & 11 & 8 \\
29 & 449 & 26 & - & 14 & 11 & 7 \\
30 & 439 & 26 & - & 14 & 11 & 7 \\
31 & 420 & 28 & - & 14 & 10 & 7 \\
32 & 409 & 26 & - & 12 & 9 & 7 \\
33 & 381 & 30 & 26 & 12 & 9 & 6 \\
34 & 352 & 31 & 25 & 12 & 7 & 6 \\
35 & 338 & 32 & 24 & 11 & 7 & 6 \\
36 & 325 & 32 & 17 & 11 & 7 & 6 \\
37 & 312 & 32 & 16 & 11 & 7 & 6 \\
38 & 292 & 35 & 16 & 11 & 7 & 5 \\
39 & 280 & 36 & 16 & 10 & 7 & 5 \\
40 & 258 & 37 & 14 & 10 & 6 & 5 \\
41 & 248 & 37 & 14 & 9 & 6 & 5 \\
42 & 238 & 37 & 12 & 9 & 6 & 5 \\
43 & 229 & 37 & 12 & 7 & 6 & 5 \\
44 & 220 & 37 & 12 & 7 & 6 & 5 \\
45 & 211 & 37 & 12 & 7 & 6 & 4 \\
46 & 203 & 37 & 11 & 7 & 6 & 4 \\
47 & 192 & 44 & 11 & 7 & 6 & 4 \\
48 & 184 & 44 & 11 & 7 & 5 & 4 \\
49 & 177 & 44 & 11 & 7 & 5 & 4 \\
50 & 170 & 44 & 11 & 7 & 5 & 4 \\
\hline & & & & & &
\end{tabular}


than a preceding eigenvalue. For every case of $p$ with anomalous solutions, the maximum value of $n$ possible (i.e., $n_{\max }$ ) before degenerate results emerged was identified.

Finally, to examine how degeneracy varied with different levels of $n$ and $p$, solutions were generated with $n=400,600,800$, and 1,000 for all values of $p$ that produced degenerate results.

\section{Results and Discussion}

Results are displayed in Table 1. No degenerate solutions occurred for values of $p$ between 5 and 15. Degenerate solutions emerged, however, for all cases in which $p$ ranged from 16 to 50 . For instance, anomalies emerged when $n$ was larger than 745 for $p=16$.

Ordinal positions of eigenvalues that marked the onset of anomalies for values of $n$ of $n_{\max }+1$, $400,600,800$, and 1,000 are also given in Table 1. For example, the 14th eigenvalue was larger than the 13th eigenvalue for $p=16$ and $n=1,000$.

Results indicate that the Allen and Hubbard procedure degenerates systematically and pervasively. Depending on parameters, anomalies may even emerge within the first $p / 2$ eigenvalues. Degeneracy in Equation 1, which may occur because of its recursive nature, is a function of both $n$ and $p$, and it is most serious when large $n$ and $p$ values are combined.

These results are consistent with previous findings of degeneracy in Equation 1 (Lautenschlager, 1989; Longman et al., 1989), and they demonstrate that using this procedure for its full range of $n$ and $p$ is inappropriate. It is recommended that researchers not use Equation 1 for any application with $p=16$ or larger and $n$ larger than the relevant $n_{\max }$. It is also recommended that alternative methods be explored for obtaining criterion eigenvalues for Horn's (1965) procedure (e.g., Lautenschlager, 1989; Longman et al., 1989).

\section{References}

Allen, S. J., \& Hubbard, R. (1986). Regression equations for the latent roots of random data correlation matrices with unities on the diagonal. Multivariate Behavioral Research, 21, 393-398.

Hays, R. D. (1987). PARALLEL: A program for performing parallel analysis. Applied Psychological Measurement, $11,58$.

Holden, R. R., Longman, R. S., Cota, A. A., \& Fekken, G. C. (1989). PAR: Parallel analysis routine for random data eigenvalue estimation. Applied Psychological Measurement, 13, 192.

Horn, J. L. (1965). A rationale and test for the number of factors in factor analysis. Psychometrika, 30 , 179-185.

Kaiser, H. F. (1960). The application of electronic computers to factor analysis. Educational and Psychological Measurement, 20, 141-151.

Lautenschlager, G. J. (1989). A comparison of alternatives to conducting Monte Carlo analyses for determining parallel analysis criteria. Multivariate Behavioral Research, 24, 365-395.

Loehlin, J. C. (1987). Latent variable models. Hillsdale NJ: Erlbaum.

Longman, R. S., Cota, A. A., Holden, R. R., \& Fekken, G. C. (1989). A regression equation for the parallel analysis criterion in principal components analysis: Mean and 95th percentile eigenvalues. $M u l-$ tivariate Behavioral Research, 24, 59-69.

Velicer, W. F., \& Jackson, D. N. (1990). Component analysis versus common factor analysis: Some issues in selecting an appropriate procedure. Multivariate Behavioral Research, 25, 1-29.

Zwick, W. R., \& Velicer, W. F. (1986). Comparison of five rules for determining the number of components to retain. Psychological Bulletin, 99, 432-442.

\section{Acknowledgments}

The authors thank two reviewers for their comments on an earlier version of this article. Preparation of this paper was supported by the Medical Research Council of Canada, the Social Sciences and Humanities Research Council of Canada, and the Ontario Ministry of Health. Portions of this paper were presented at the annual meeting of the Psychometric Society (July, 1989) in Los Angeles CA, U.S.A.

\section{Author's Address}

Send requests for reprints or further information to G. Cynthia Fekken, Department of Psychology, Queen's University, Kingston, Ontario, Canada, K7L 3 N6. 